\title{
Reciprocal allogrooming among unrelated Norway rats (Rattus norvegicus) is affected by previ- ously received cooperative, affiliative and aggressive behaviours
}

\author{
Binia Stieger ${ }^{1,2}$, Manon K. Schweinfurth ${ }^{1}$ and Michael Taborsky ${ }^{1}$ \\ ${ }^{1}$ Institute of Ecology and Evolution, University of Bern, Wohlenstrasse 50a, \\ CH-3032 Hinterkappelen, Switzerland \\ ${ }^{2}$ Correspondence: binia.stieger@iee.unibe.ch/phone: +41 316319151
}

Reciprocity can generate stable levels of cooperation among unrelated social partners. If individuals interact repeatedly, costs of altruistic acts can be compensated through an exchange of donor and receiver roles. Frequent interactions are conducive to attaining evolutionarily stable reciprocal exchange. High interaction frequencies are typical for group members maintaining close relationships among one another, which may thereby facilitate reciprocity. Norway rats (Rattus norvegicus) are highly social animals that were experimentally shown to reciprocally exchange food donations and allogrooming. Here we tested experimentally the relationship between reciprocal cooperation and other social behaviours exchanged within dyads of wild-type Norway rats. In particular, we asked whether and how interactions differing in quality (characterised by affiliative and aggressive behaviours) influence reciprocal exchanges of different social services. Our experiment involved three steps: Focal individuals experienced social partners that were either providing them with food or not, via a learnt stick-pulling task. Thereafter, they could either interact physically with these partners, or not. Subsequently, we induced allogrooming among them by applying saltwater to an inaccessible part of the body, and tested for the reciprocation of allogrooming. When individuals were allowed to interact freely, previously cooperative food providers exhibited more aggression towards focal individuals than previously uncooperative partners, which might reflect an attempt to coercively demand a return of food provisioning from focal rats. Higher frequencies of affiliative behaviours and lower frequencies of aggressive behaviours experienced during the unrestricted interaction phase tended to increase the focal rats' propensity to engage in grooming the partner in the subsequent induced allogrooming 
phase. This suggests that affiliative and aggressive behaviours affect the allogrooming propensity of rats. In particular, higher frequencies of received aggression decreased the propensity to reciprocate previously received cooperation. We provide experimental evidence that rats are more likely to groom partners that pulled a stick to deliver food to them. Reciprocal exchange of allogrooming depends apparently on experienced cooperation, but also on the quality of the social relationship.

\section{Significance Statement}

Close social relationships among individuals may enhance reciprocal exchange of services and thereby ensure long-term cooperation. Thus we tested whether in unrelated and previously unfamiliar Norway rats the quality of social interactions, that is, the amount of exchanged affiliative and aggressive behaviours, affects reciprocal cooperation, and whether received cooperation in turn predicts subsequent social behaviour. Our results show that focal individuals are generally more helpful to previously helpful partners, but that the quality of social interactions may modify their decision to cooperate. Received aggressive and affiliative behaviours affected the subsequent reciprocal exchange of hygienic behaviour. Moreover, received food provisioning affected the exchange of cooperative, affiliative and aggressive behaviours also outside of a food-provisioning context. These data reveal a close relationship between the exchange of social behaviours between individuals and their propensity to cooperate with one another.

Keywords: Norway rats, cooperation, reciprocal altruism, allogrooming, food provisioning, social bonds 


\section{Introduction}

Behaviours by which an actor benefits a receiver while paying immediate costs are referred to as altruistic (Wilson 1975). The establishment of evolutionarily stable levels of altruism among non-kin can be explained for instance by coercion (Tebbich et al. 1996; Fehr and Gächter 2002) or reciprocity (Trivers 1971; Axelrod and Hamilton 1981), or by a combination of both (Quiñones et al. 2016). Whilst being coerced to cooperate, individuals help others in order to avoid being punished (Clutton-Brock and Parker 1995; Raihani et al. 2012), with the mere threat of being punished sometimes sufficing to enforce cooperation (Cant 2011). This may occur, for instance, in the context of allogrooming. Allogrooming can be an altruistic behaviour when a donor benefits a recipient at own costs, which may involve the loss of saliva and electrolytes (Ritter and Epstein 1974), opportunity and vigilance costs (Ritter and Epstein 1974; Mooring and Hart 1995; Viblanc et al. 2011; reviewed in Hawlena et al. 2007). Potential benefits for receivers of allogrooming include the removal of ectoparasites (Tanaka and Takefushi 1993) and the receipt of antimicrobial substances (Hughes et al. 2002). However, allogrooming may also be enforced from subordinates by dominant individuals subjecting them to high amounts of aggression (Silk 1982). In this case, lower ranked individuals are coerced to groom dominant individuals to avoid aggression, which can be reflected by a positive correlation between aggression received and grooming provided (Schino et al. 2005). On the other side of the coin, by pursuing a "grooming up the hierarchy" strategy (Seyfarth 1977) low ranked individuals may reduce the amount of aggression received from dominant individuals when frequently grooming them. Thus, allogrooming can be a means of reducing exposure to aggression, which may cause a negative correlation between grooming provided and aggression received (Seyfarth 1977; Silk 1982; Gumert and Ho 2008). Besides reduced aggression (Gumert and Ho 2008), numerous other benefits may be provided by a dominant partner in return for being groomed, including agonistic support (Seyfarth and Cheney 1984; Koyama et al. 2006), increased social tolerance (Ventura et al. 2006), and help in obtaining access to mating partners (Stopka and Macdonald 1999). 
Alternatively, reciprocal altruism can lead to evolutionarily stable levels of cooperation by compensating costs of an altruistic act through a systematic exchange of roles between donors and recipients (Trivers 1971). In general, the decision to reciprocate favours is influenced by the donor's cost of helping (Schneeberger et al. 2012), the benefit to the receiver (Wilkinson 1984; Dolivo and Taborsky 2015a), and the probability of receiving help in return (Lehmann and Keller 2006; Taborsky et al. 2016). The latter is a function of the frequency of interactions in which help can be exchanged. Thus, frequent interactions facilitate the establishment and maintenance of reciprocal exchanges (Barta et al. 2011). The relationship model is often considered to explain cooperation and social relationships in primates (Dubar 1988; Silk et al. 2006). It assumes that social interactions such as allogrooming help to establish and maintain close social relationships. Resulting 'social bonds' characterize close amicable relationships between pairs of individuals that may occur among mating pairs, parents and offspring or other types of relations, including unrelated individuals (Kutsukake and Clutton-Brock 2006; Cameron et al. 2009; Wittig et al. 2014; reviewed in Seyfarth and Cheney 2012). Such relationships, which also have been referred to as "friendships" (Silk 2002, p. 434), are conducive to high degrees of reciprocity in directional, nonaggressive activities such as mutual grooming and food sharing (Silk 2002). For example, female vampire bats share blood more often with familiar individuals with which they form stable cooperative relationships than with unfamiliar individuals (Wilkinson 1984; Carter and Wilkinson 2013, 2016). Reciprocal cooperation among bonded individuals has been observed also in primates and birds (Schino and Aureli 2008; St-Pierre et al. 2009). In turn, high levels of cooperation among social partners may reinforce their social bond (Gill 2012). Several studies, however, did not find that individuals with a higher relationship quality show higher initial investments in cooperation (Barrett et al. 2000; Fruteau et al. 2011; Kaburu and Newton-Fisher 2016; Newton-Fisher and Kaburu 2017). This has been attributed to the possibility that social interactions may rather follow economical decision rules and biology market conditions, where the immediate social context instead of established relationships may be of primary importance. 
Here, we attempt to clarify the potential connection between cooperation and social behaviours in wild-type Norway rats (Rattus norvegicus). Although there is no evidence that female Norway rats form social bonds (Schweinfurth et al. 2017a), we aim to elucidate whether and in which way reciprocity may be influenced by the outcome of social encounters. Rats live in colonies of up to 200 individuals (Telle 1966), and recognize conspecifics individually (Gheusi et al. 1997). They show a wide range of amicable social behaviours like joint huddling, food sharing and allogrooming (Barnett and Spencer 1951). Experimentally, rats have been shown to reciprocate food donations in a food exchange task, where individuals could bring a baited platform into the reach of their partner (Rutte and Taborsky 2007, 2008), and to reciprocate allogrooming (Schweinfurth et al. 2017b).

We investigated whether focal individuals show different behaviours towards cooperative food providers compared to uncooperative non-providers. Further, we scrutinised whether the amount of reciprocal exchange is influenced by the relationship of individuals among each other, measured as exchanged affiliative and aggressive behaviours. Our experiment followed a full-factorial design, including three phases to which all focal rats were exposed. During the food-provisioning phase, focal individuals experienced either a cooperative partner providing food to them via a trained stick-pulling task, or an uncooperative partner not providing such service. In the unrestricted interaction phase, focal individuals could either freely interact with their partners, or they were prevented from direct physical contact by separation. In the induced allogrooming phase, we elicited allogrooming by applying saltwater on the focal individual's neck, and quantified the resulting grooming behaviour.

When we allowed a partner to provide food to the focal rat by pulling a stick during the food-provisioning phase, we expected focal rats to show less aggression and more affiliation towards that partner during the unrestricted interaction phase. If in turn the exchange of affiliative behaviours would influence the propensity to cooperate, we predicted rats to groom partners more often with which they exchanged more affiliative behaviours. Finally, we predicted reciprocity to be more pronounced among individuals that exchanged more affiliative and less aggressive behaviours. 


\section{Methods}

Study subjects

We used outbred wild-type adult female Norway rats (source: Animal Physiology Department, University of Groningen, Netherlands), which were individually marked by ear punching. We housed them in cages $(80 \mathrm{~cm} / 50 \mathrm{~cm} / 37.5 \mathrm{~cm}$, enriched with wooden huts, shelves, plastic tubes, litter, cardboard loo rolls, and hay as nesting material) in groups of five littermates. The ambient temperature was $20^{\circ} \mathrm{C} \pm$ $1^{\circ} \mathrm{C}$, with a relative humidity of $40-60 \%$ and a $12: 12 \mathrm{~h}$ light:dark cycle with lights on at 20:00 hours and 30 minutes of dawn and dusk. Because rats are nocturnal (Becker 1978) and are insensitive to red light, we conducted all experiments during the dark phase under red light during the day. We provided water and food (conventional rat pellets) ad libitum. Additionally, we provided either grains mix or fresh vegetables each day. Because all rats had been handled from early age on, they were well habituated to handling and the presence of an observer before and during experiments (cf. Rutte and Taborsky 2007).

\section{Pre-experimental training (food provisioning phase)}

The experimental setup was based on a two-player sequential food-exchange task (de Waal and Berger 2002; Rutte and Taborsky 2007). Test cages $(80 \times 50 \times 37.5 \mathrm{~cm})$ were divided into two equally sized chambers by a wire mesh. All focal and partner rats experienced the following trainings prior to the experiment. First, every single rat was trained to pull a stick that was connected to a movable platform in order to receive a food item (one oat flake). After each pull of a rat, the experimenter retreated the platform out of the cage. This was repeated over several training sessions, thereby teaching the rats to pull the stick in order to reach the reward. In the second part of the training, a sister was placed in the neighbouring cage compartment. From now on, the rats never received a reward by themselves for pulling the platform. Instead, they experienced that only their partner received a treat if they pulled the platform. The roles between donor and recipient were regularly exchanged, and the intervals between these alternations were increased gradually from switching the roles after each pull to switching the roles after series of pulls lasting up to seven minutes, which corresponds to the duration 
of the subsequent experimental period (see Dolivo and Taborsky 2015a for a detailed plan of the training scheme).

\section{Experimental set-up}

The experiment consisted of a sequence of three phases (Fig. 1). In the first phase (food-provisioning phase), the focal rats experienced a cooperative partner providing food to them, or an uncooperative partner providing no food. In the second phase (unrestricted interaction phase), the focal individuals could freely interact repeatedly in six subsequent trials with both partners, or they were prevented from physical contact by a separating wire mesh. In the third phase (induced allogrooming phase), we measured the reciprocal exchange of allogrooming bouts. Each focal individual $(n=19)$ received all four treatments, differing in the partner's cooperation level and possibility for physical contact with four different partners, in a randomised sequence (Fig. 1). Focal rats experienced two partners per day in a random order. We selected partners randomly, but for possible individual effects, we included their identity as a random factor in the statistical models.

During the food-provisioning phase, focal rats experienced a cooperative food-providing partner and an uncooperative non-providing partner on each of four consecutive days. The cooperative partner provided several food items (oat flakes), one at a time, during seven minutes. The number of food items was not fixed by the experimenter but by the pulling propensity of the donating rat. The pulling mechanism was blocked in the uncooperative treatment so that the focal rat did not receive any food from the partner. The amount of food provided by the cooperative partner did not influence future cooperative behaviour (GLMM; $6=-0.019 \pm 0.026, z=-0.722, p=0.47$ ), which is why this variable was not included in further statistical analyses.

In the subsequent unrestricted interaction phase, we placed the focal individual and either the cooperative or uncooperative partner in an arena (glass tank: $80 \mathrm{~cm} / 40 \mathrm{~cm} / 40 \mathrm{~cm}$ ). Here, they could freely interact for one hour, on six consecutive days. In the control treatments, focal rats were physically separated from their partner by a wire mesh. We video recorded the six encounters (camera: Sony 
HDR-CX550VE; night vision-mode) and analysed the frequencies of different social behaviours (affiliative and aggressive behaviours, see Table 1). In the following induced allogrooming phase, we measured the reciprocal exchange of grooming bouts. We placed the focal individual and the respective partner in the same arena as used in the unrestricted interaction phase. We applied saltwater (250g salt/I) onto the neck of both rats, which is an established method to induce allogrooming in rats (Schweinfurth et al. 2017b). We recorded the grooming frequency (counts) of both rats for $20 \mathrm{~min}$.

Video analyses; We analysed the recorded video material from the unrestricted interaction (1h per trial) and induced allogrooming (20 min per trial) phases using the freeware observer program Solomon Coder (version: beta 14.03.10). During the unrestricted interactions, we focussed on affiliative and aggressive behaviours (see Table 1). Additionally, we recorded which individual initiated the aggressive interactions. We quantified the exchange of allogrooming bouts while we induced allogrooming. Self-grooming and allogrooming correlated positively with each other (Pearson's correlation test, $t=2.46, n=19, r=0.17, p=0.015)$, hence we combined these two behaviours (called "affiliation"). Depending on the analysis, single behaviours of either category were summed up and analysed either as total amounts shown during the unrestricted interaction phase or as sums over each day of the unrestricted interaction phase. To minimize observer bias, the experimenter was blind to the treatments while analysing the videos.

\section{Statistical Analysis}

We analysed the data using the R (version 3.2.4) packages "Ime4", "MASS" and "car". Two individuals died of old age during the experiment, which is why the dataset on these two rats was not complete. Furthermore, one video recording of the induced allogrooming phase was lost due to technical failure.

\section{Influence of previous food provisioning and physical contact on grooming provided}

To test whether food provisioning and the possibility for physical contact influenced the grooming behaviour in the induced allogrooming phase, we used a linear mixed effects model (LM). The number of grooming bouts by focal rats in the induced allogrooming phase was not normally distributed 
(Shapiro-Wilk test: $W=0.95, p=0.006)$. We thus transformed the data according to Box and Cox (1964), with an optimal $\lambda$ of 0.6 . We included the transformed data of the frequency of given grooming bouts by focal rats in the induced allogrooming phase as the response variable. We used experienced cooperation during the food-provisioning phase (cooperative vs. uncooperative partner), possibility for physical contact during the unrestricted interaction phase (interacting vs. separated), and frequency of received grooming bouts by the partner during the induced allogrooming phase as explanatory variables. To account for multiple testing of focal rats and the possible influence of the partner's identity, we included both individual identities as random effects. The interaction between received food provisioning, possibility for physical contact and received grooming bouts was not significant (LM; $B=-$ $0.16 \pm 0.36, t=-0.41, p=0.676$ ) and was therefore excluded from the final model (Engqvist 2005).

Influence of previous food provisioning on cooperative, affiliative and aggressive behaviours To test whether the number of exchanged grooming bouts between focal rats and partners differed between treatments, we applied a Wilcoxon matched-pairs signed-ranks test where we compared given and received grooming bouts by focal rats with previously cooperative and uncooperative partners. To assess reciprocity in allogrooming bouts, we used a GLMM assuming a Poisson distribution with log-link. We included the sum of frequencies of given grooming bouts by focal rats across the six days of the unrestricted interaction phase as the response variable. We used the sum of frequencies of received grooming bouts by the partner across the six days of the unrestricted interaction phase and experienced cooperation during the food-provisioning phase (cooperative vs. uncooperative partner) as explanatory variables. To account for multiple testing of focal rats and the potential influence of the partner's identity, we included both individual identities as random effects. We checked the model for overdispersion, which was not the case. We used a non-parametric test because our response variable was not normally distributed (Shapiro-Wilk test: $W=0.92, p=0.014$ ). We used Spearman rank correlation analyses to assess the relationship between given and received grooming bouts separately for both treatments. 
To test whether focal individuals differ in their behaviour between cooperative and uncooperative partners during the unrestricted interaction phase, we used linear mixed effects models (LM). Neither affiliative (Shapiro-Wilk test: $W=0.95, p<0.001$ ) nor aggressive behaviours (Shapiro-Wilk test: $W=$ $0.43, p<0.001$ ) were normally distributed. We thus transformed the data according to Box and Cox (1964), with an optimal $\lambda$ of 0.512 for affiliative, and -0.698 for aggressive behaviours. As the response variables, we included the transformed data either for affiliative or aggressive behaviours shown by focal individuals towards their partner. As explanatory variables, we used experienced cooperation during the food provisioning phase (cooperative vs. uncooperative partner) and day (day 1 to 6 of the unrestricted interaction phase) as covariate. Focal and partner rat's identities were included as random effects. In both models, the interaction between experienced cooperation and day was not significant (affiliative behaviours: $\mathrm{LM}, 6=0.01 \pm 0.01, t=0.10, p=0.917$; aggressive behaviours: $\mathrm{LM}, b=-0.001 \pm$ $0.02, t=-0.06, p=0.951)$, hence it was excluded from the final models (Engqvist 2005).

In contrast to affiliative behaviours, our results revealed a significant influence of the treatment on the aggression by focal rats (see Results). Therefore, we only further analysed the data on aggression by partners but not the data on affiliative behaviours. To analyse whether focal individuals or their partners initiated aggression more often during the unrestricted interaction phase across treatments, we used a Pearson's $\chi^{2}$-test. In contrast to aggression shown by focal rats, we could not successfully transform the data of the partner's aggression to accord with a normal distribution. We therefore used a Wilcoxon matched-pairs signed-ranks test to check whether the amounts of aggression exhibited towards focal individuals differed between cooperative food providers and uncooperative nonproviders. In addition, we applied a Spearman signed-ranks correlation test to assess whether the amount of aggression exhibited by focal rats correlated with the amount of received aggression by their social partners. 
Influence of previous cooperative, affiliative and aggressive behaviours on grooming provided To test whether behaviours in the unrestricted interaction phase influenced the behaviour in the induced allogrooming phase, we used a GLMM. The response variable was the frequency of grooming bouts performed by focal rats in the induced allogrooming phase. We used affiliative and aggressive behaviours shown by partners in the unrestricted interaction phase as explanatory variables, and identity of focal rats and their partners as random effects. Here, we were not interested in the interaction between affiliative and aggressive behaviours and therefore did not include it in our model. To test whether behaviours in the unrestricted interaction phase and the treatments influenced the reciprocal exchange of grooming bouts in the induced allogrooming phase, we used an additional LM. We calculated the ratio between given and received grooming bouts by dividing the number of grooming bouts provided by focal individuals to their partner by the number of grooming bouts focal rats received from this partner. Because this ratio of given and received grooming bouts was not normally distributed (Shapiro-Wilk test: $W=0.743, p<0.001$ ), we log-transformed this variable to accord with a normal distribution. The response variable was hence the log transformed ratio of grooming bouts by focal rats and received grooming bouts by partner rats in the induced allogrooming phase. We included treatment, affiliative and aggressive behaviours shown by partners in the unrestricted interaction phase as explanatory variables, and identity of focal rats and their partners as random effects. Because means of affiliative and aggressive behaviours strongly deviated from zero, we mean-centred the data by subtracting the means of each behavioural category. Because we were not interested in the interaction between treatment, affiliative and aggressive behaviours, we did not include it in our model. 


\section{Results}

Influence of previous food provisioning and physical contact on grooming provided

In the induced allogrooming phase, focal rats groomed previously cooperative food providers more often than previously uncooperative partners ( $L M ; B=0.48 \pm 0.19, t=2.53, p=0.018$, Fig. 2a). This effect did not differ between experimental pairs that were enabled or prevented from interacting with each other ( $L M ; B=-0.15 \pm 0.36, t=-0.41, p=0.676)$. Importantly, received grooming bouts during the induced allogrooming phase increased the number of grooming bouts given by focal individuals (LM; $B=0.08 \pm 0.03, t=2.60, p=0.010$, Fig. $2 b)$. Furthermore, focal rats groomed those partners more often with which they previously could not interact compared to when physical contact was possible (LM; $B=-0.50 \pm 0.18, t=-2.74, p=0.008$, Fig. 2a).

Influence of previous food provisioning on cooperative, affiliative and aggressive behaviours During the unrestricted interaction phase, grooming bouts by both, partners and focal rats, did not differ between previously cooperative and uncooperative individuals (Wilcoxon test, given: $W=140.5$, $n=19, p=0.885$; received: $W=159.5, n=19, p=0.407)$. However, focal rats reciprocated grooming bouts with previously cooperative rats more readily than with previously uncooperative partners (GLMM; interaction between frequency of received grooming bouts and cooperativeness of partners: $b=0.02 \pm 0.01, z=1.98, p=0.048)$, even though the relationship between given and received allogrooming was positive in both treatments (significantly positive with previously cooperative partners: Spearman rank correlation, $S=412.51, n=19, r=0.494, p=0.044$; positive by trend with previously uncooperative partners: Spearman rank correlation, $S=367.08, n=19, r=0.460, p=0.073$ ).

During the six days of the unrestricted interaction phase, in both treatments the initiation of aggression did not differ between focal rats and their partners (Pearson's $\chi^{2}$-test, $\chi^{2}=2.120, d f=1, p=0.145$ ). Partners that were able to provide food were more aggressive towards focal individuals compared to the partners that could not provide food (food providers: $\mu=9.16 \pm 3.21$ aggressive events per $1 \mathrm{~h}$ of 
physical interaction; non-food providers: $\mu=3.82 \pm 1.72$ aggressive events per $1 \mathrm{~h}$ of physical interaction; Wilcoxon matched-pairs signed-ranks test, $V=1409.5, n=19, p<0.001)$. The amount of aggression exhibited by focal individuals correlated positively with aggression received from their partners (Spearman test, $S=469410, n=19, r=0.762, p<0.001$ ). Hence, focal rats directed more aggressive behaviours to previously experienced cooperative food providers, which attacked them more often in the unrestricted interaction phase, than towards previously uncooperative partners, which attacked them less often (cooperative food providers: $\mu=1.19 \pm 0.96$; uncooperative partners: $\mu=0.50 \pm 0.91$; $\mathrm{LM}, 6=-0.09 \pm 0.04, t=-2.28, p<0.001)$. In contrast, the levels of affiliative behaviours shown by focal individuals towards previously cooperative or uncooperative partners did not differ ( $L M, b=0.18 \pm$ $0.17, t=1.06, p=0.310)$. Affiliative behaviour did not change over time, however aggressive behaviours decreased over the six days of the unrestricted interaction phase (LM, affiliative: $B=-0.02 \pm 0.05, t=-$ $0.53, p=0.595$; aggressive: $\mathrm{LM}, b=-0.01 \pm 0.01, t=-1.18, p<0.001)$.

Influence of previous cooperative, affiliative and aggressive behaviours on grooming provided Affiliative behaviours performed by partners during the unrestricted interaction phase tended to increase the focal rats' grooming frequency during the induced allogrooming phase, although this relationship was not significant (GLMM, $6=0.005 \pm 0.003, z=1.68, p=0.093$, Fig. 4a). In contrast, received aggression during the unrestricted interaction phase yielded reduced grooming frequencies of focal rats in the induced allogrooming phase (GLMM, $6=-0.004 \pm 0.002, z=-1.96, p=0.050$, Fig. 4b). The ratio of grooming bouts provided by focal individuals to partners and grooming bouts received from partners, as a measure of immediate reciprocation, was neither influenced by the treatment $(\mathrm{LM}, \mathrm{B}=$ $-0.075 \pm 0.258, t=0 .-0.29, p=0.759)$, nor by affiliative behaviours shown by partners (LM, $b=-0.004 \pm$ $0.004, t=-0.95, p=0.326)$. Nevertheless, aggression displayed by partners decreased this ratio, i.e. focal rats provided more aggressive partners with fewer grooming bouts per allogrooming bouts received from them $(\mathrm{LM}, b=-0.004 \pm 0.002, z=-2.22, p=0.024)$. 


\section{Discussion}

In this study we investigated the role of cooperation on affiliative and aggressive social interactions and vice versa using wild-type Norway rats. Our data suggest that previous aggression may reduce the allogrooming propensity in subsequent interactions, whereas the previous exchange of affiliative behaviours may rather increase the probability that this behaviour is performed. Contrary to our predictions, when rats were prevented from physical contact they responded with elevated grooming levels in the subsequent experimental phase, compared to dyads that were enabled to physically interact.

We predicted that rats would show more affiliative and less aggressive behaviours towards previous cooperative food providers than towards previous uncooperative partners. However, previously cooperative partners of focal rats showed elevated levels of aggression toward focal individuals in the unrestricted interaction phase of the experiment compared to previously uncooperative partners. This is probably the reason why focal individuals also showed more aggressive behaviour towards previously cooperative partners than to previously uncooperative ones, even though the aggression rates of focal individuals towards their partners were generally low (0.5-1.19 events/hour). We do not know why partner rats that served as cooperators in the first phase of the experiment attacked focal rats more often in the subsequent unrestricted interaction phase, but one reason might have been belied expectation to receive something back in return for the previously made food donations. Hence such aggression may have reflected frustration or coercion. Previous results somewhat support this interpretation. In a study with laboratory rats, aggressive behaviour was enhanced in situations with nonreward under conditions in which the rats expected a reward (Gallup 1965). A study with wild-type Norway rats showed that aggression may be used to enforce help from reluctant experimental partners (Dolivo and Taborsky 2015b).

In accordance with a previous study investigating the propensity of unrelated Norway rats to form social bonds (Schweinfurth et al. 2017a), our results do not hint at social bond formation in female Norway rats. Participation in frequent socio-positive interactions (Silk 2002; Massen et al. 2010) were 
not observed in our study among focal individuals and their previously experienced cooperative partners. Additionally, aggression was shown in all dyads and aggressive and affiliative behaviours did not change over time, suggesting no bond formation. Although social bonds may facilitate altruism (Melis et al. 2006; Schino and Aureli 2009; St-Pierre et al. 2009), Norway rats cooperate reciprocally based solely on received help in preceding interactions (Dolivo et al. 2016), comparable to dogs in the study of Gfrerer and Taborsky (2017). Similarly, grooming decisions within dyads of wild male chimpanzees are apparently not based on trust or bonds, whereas the immediate social context and the influence of third parties are important factors influencing cooperative behaviours (Kaburu and Newton-Fisher 2016). Besides reciprocity, aggression may be an additional tool for generating altruistic help (Dolivo and Taborsky 2015b). Likewise, our data suggest that immediate behaviours, such as affiliation and aggression, may affect the decision to cooperate. In natural Norway rat colonies, mark-recapture studies revealed high fluctuations in population size, between 2 and 100 individuals within one year (McGuire et al. 2006). Such unstable social situation might favour reciprocal cooperation based on recent interactions rather than long-term social bonds.

We predicted that dyads sharing more affiliative rather than aggressive behaviours in the unrestricted interaction phase to cooperate more readily in the subsequent induced allogrooming phase, which indeed tended to be the case. Contrary to our predictions, focal rats groomed partners with whom they had not physically interacted before more often than those with whom they had previous physical contact. Focal rats may have shown elevated levels of allogrooming because of its function as an appeasement gesture while interacting with a new partner (Kutsukake and Clutton-Brock 2006). Additionally, exposure to a novel artificial environment enhances self-grooming (Jolles et al. 1979) and reduces aggression in rats (Albonetti and Farabollini 1996). Whether this might have influenced the behaviour of rats in our experiment is presently not clear. However, all rats showed at least some aggression towards their partner during the unrestricted interaction phase, and aggression is known to influence cooperation propensity (Madden and Clutton-Brock 2009; Carne et al. 2011; Dolivo and 
Taborsky 2015b). Thus, if rats were prevented from physical contact, they were also not exposed to aggression, which may have caused the subsequently higher amounts of exchanged allogrooming.

In our experiment, cooperative food providers received more allogrooming bouts than uncooperative partners even after six days of separation. However, infrequent interactions are expected to reduce the likelihood to reciprocate favours among social partners (Trivers 1971; Axelrod and Hamilton 1981; Taborsky et al. 2016) and it has been argued that temporal discounting limits reciprocal cooperation over long time intervals (e.g. Stephens et al. 2002, 2005). In particular, rats were shown to devalue rewards within a matter of seconds (Richards et al. 1997), and hence it was expected that reciprocal cooperation should be restricted to short time intervals (Stevens and Hauser 2004). Nevertheless, our data show that Norway rats can reciprocate received help after a delay of six days (see also Schino and Aureli 2010). Furthermore, rats rewarded previous food providers with increased allogrooming levels, which is a different commodity. This confirms results from a recent study demonstrating commodity trading in Norway rats (Schweinfurth and Taborsky 2017).

We predicted reciprocity to be fostered by affiliative relationships. Indeed, in the unrestricted interaction phase given and received grooming bouts by focal individuals correlated positively only when interacting with previously cooperative partners. Rats provide help according to the amount and quality of help they received (Rutte and Taborsky 2008; Dolivo and Taborsky 2015a), which is in accordance with our data. Furthermore, reciprocity was less pronounced among pairs with many aggressive interactions. Focal individuals provided aggressive individuals with proportionally less grooming bouts per received allogrooming. Dolivo and Taborsky (2015b) also found that aggression exhibited by previously cooperative partners reduced the propensity of rats to reciprocate received help. However, in their study aggression exhibited by previously uncooperative partners increased helping levels, suggesting coercion of help. 
Our data show that in Norway rats, the cooperativeness of individuals influences their partners' behaviours and the quality of interactions, and vice versa. While affiliation tends to increase the propensity to provide allogrooming, aggression rather reduces it. We did not find evidence for social bonds but rather that preceding interactions directly influenced the reciprocal exchange of commodities, even if this happened after an intermission of several days. This supports the notion that temporal discounting does not limit reciprocal cooperation in Norway rats.

\section{Acknowledgments}

We thank Leif Engqvist for help with data analyses, Karin Schneeberger, Leif Engqvist and two anonymous referees for helpful comments on a previous version of the manuscript, Evi Zwygart for care of the rats, Markus and Bea Wymann for technical support, and Valentina Balzarini and Michelle Gygax for drawings. Funding was provided by a Schweizerischer Nationalfonds (SNF)-grant 31003A_156152 to MT.

\section{Conflict of Interest}

The authors declare that they have no conflict of interest.

\section{Ethical approval}

All applicable international, national, and institutional guidelines for the care and use of animals were followed. The housing of the animals and the experimental procedure were authorized by the Swiss Federal Veterinary Office under license BE98/11. During the experiment no injuries occurred. In addition, the animals were constantly monitored during all experiments, and if any deviant behaviour or unexpected physical reactions had occurred, the experiments would have been immediately stopped. 


\section{Data availability}

The datasets generated and analysed during the current study are available from the corresponding author on request. 


\section{References}

Albonetti ME, Farabollini F (1996) Differential effects of restraint and novelty on the social behaviour of female rats. Behav Process 37:209-215

Axelrod R, Hamilton WD (1981) The evolution of cooperation. Science 211:1390-1396

Barnett SA, Spencer MM (1951) Feeding behaviour and interspecific competition in wild rats. Behaviour 3:229-242

Barrett L, Henzi SP, Weingrill T, Lycett JE, Hill RA (2000) Female baboons do not raise the stakes but they give as good as they get. Anim Behav 59:763-770

Barta Z, McNamara JM, Huszár DB, Taborsky M (2011) Cooperation among non-relatives evolves by state-dependent generalized reciprocity. Proc R Soc Lond B 278:843-848

Becker K (1978) Rattus norvegicus (Berkenhout 1769) - Wanderratte. In: Niethammer J, Krapp F (eds) Handbuch der Säugetiere Europas. Aula-Verlag, Wiesbaden, pp 401-420

Box GEP, Cox DR (1964) An analysis of transformations. J R Stat Soc B 26:211-252

Cameron EZ, Setsaas TH, Linklater WL (2009) Social bonds between unrelated females increase reproductive success in feral horses. P Natl Acad Sci USA 106:13850-13853

Cant MA (2011) The role of threats in animal cooperation. Proc R Soc Lond B 278:170-178

Carne C, Wiper S, Semple S (2011) Reciprocation and interchange of grooming, agonistic support, feeding tolerance, and aggression in semi-free-ranging barbary macaques. Am J Primatol 73:11271133

Carter GG, Wilkinson GS (2013) Food sharing in vampire bats: reciprocal help predicts donations more than relatedness or harassment. Proc R Soc B 280:20122573

Clutton-Brock TH, Parker GA (1995) Punishment in animal societies. Nature 373:209-216

de Waal FBM, Berger ML (2000) Payment for labour in monkeys. Nature 404:563-563

Dolivo V, Rutte C, Taborsky M (2016) Ultimate and proximate mechanisms of reciprocal altruism in rats. Learn Behav 44:223-226

Dolivo V, Taborsky M (2015b) Cooperation among Norway rats: the importance of visual cues for reciprocal cooperation, and the role of coercion. Ethology 121:1071-1080

Dolivo V, Taborsky M (2015a) Norway rats reciprocate help according to the quality of help they received. Biol Lett 11:20140959

Dunbar RIM (1988) Primate social systems. Croom Helm, London

Engqvist L (2005) The mistreatment of covariate interaction term in linear model analyses of behavioural and evolutionary ecology studies. Anim Behav 70:967-971

Fehr E, Gächter S (2002) Altruistic punishment in humans. Nature 415:137-140

Fruteau C, Lemoine S, Hellard E, van Damme E, Noë R (2011) When females trade grooming for grooming: testing partner control and partner choice models of cooperation in two primate species. Anim Behav 81:1223-1230 
Gallup GG Jr (1965) Aggression in rats as a function of frustrative nonreward in a straight alley. Psychon Sci 3:99-100

Gfrerer N, Taborsky M (2017) Working dogs cooperate among one another by generalised reciprocity. Sci Rep 7:43867

Gheusi G, Goodall G, Dantzer R (1997) Individually distinctive odours represent individual conspecifics in rats. Anim Behav 53:935-944

Gill SA (2012) Strategic use of allopreening in family-living wrens. Behav Ecol Sociobiol 66:757-763

Gumert MD, Ho M-HR (2008) The trade balance of grooming and its coordination of reciprocation and tolerance in indonesian long-tailed macaques (Macaca fascicularis). Primates 49:176-185

Hawlena H, Bshary D, Abramsky Zvika, Krasnov BR (2007) Benefits, costs and constraints of antiparasitic grooming in adult and juvenile rodents. Ethology 113:394-402

Hughes WHO, Eilenberg J, Boomsma JJ (2002) Trade-offs in group living: transmission and disease resistance in leaf-cutting ants. Proc R Soc Lond B 269:1811-1819

Jolles J, Rompa-Barendregt J, Gispen WH (1979) Novelty and grooming behavior in the rat. Behav Neural Biol 25:563-572

Kaburu SSK, Newton-Fisher NE (2016) Bystanders, parceling, and an absence of trust in the grooming interactions of wild male chimpanzees. Sci Rep 6:20634

Koyama NF, Caws C, Aureli F (2006) Interchange of grooming and agonistic support in chimpanzees. Int J Primatol 27:1293-1309

Kutsukake N, Clutton-Brock TH (2006) Social functions of allogrooming in cooperatively breeding meerkats. Anim Behav 72:1059-1068

Lehmann L, Keller L (2006) The evolution of cooperation and altruism - a general framework and a classification of models. J Evol Biol 19:1365-1376

Madden JR, Clutton-Brock TH (2009) Manipulating grooming by decreasing ectoparasite load causes unpredicted changes in antagonism. Proc R Soc Lond B 276:1263-1268

Massen JJM, Sterck EHM, de Vos H (2012) Close social associations in animals and humans: functions and mechanisms of friendship. Behaviour 146:1379-1412

McGuire B, Pizzuto T, Bemis WE, Getz LL (2006) General ecology of a rural population of Norway rats (Rattus norvegicus) based on intensive live trapping. Am Midl Nat 155:221-236

Melis AP, Hare B, Tomasello M (2006) Engineering cooperation in chimpanzees: tolerance constraints on cooperation. Anim Behav 72:275-286

Mooring MS, Hart BL (1995) Costs of allogrooming in impala: distraction from vigilance. Anim Behav 49:1414-1416

Newton-Fisher NE, Kaburu SK (2017) Grooming decisions under structural despotism: the impact of social rank and bystanders among wild male chimpanzees. Anim Behav 128:153-164

Quiñones AE, van Doorn S, Pen I, Weissing FJ, Taborsky M (2016) Negotiation and appeasement can be more effective drivers of sociality than kin selection. Philos T Roy Soc B 371:1687 
Raihani NJ, Thornton A, Bshary R (2012) Punishment and cooperation in nature. Trends Ecol Evol 27:288-295

Richards JB, Mitchell SH, de Wit H, Seiden LS (1997) Determination of discount functions in rats with an adjusting-amount procedure. J Exp Anal Behav 67:353-366

Ritter RC, Epstein AN (1974) Saliva lost by grooming: a major item in the rat's water economy. Behav Biol 11:581-585

Rutte C, Taborsky M (2007) Generalized reciprocity in rats. PLoS Biol 5:e196

Rutte C, Taborsky M (2008) The influence of social experience on cooperative behaviour of rats (Rattus norvergicus): direct vs generalised reciprocity. Behav Ecol Sociobiol 62:499-505

Schino G, Aureli F (2008) Grooming reciprocation among female primates: a meta-analysis. Biol Lett 4:9-11

Schino G, Aureli F (2009) Reciprocal altruism in primates: partner choice, cognition and emotions. Adv Stud Behav 39:45-69

Schino G, Aureli F (2010) A few misunderstandings about reciprocal altruism. Comun Integr Biol 3:561563

Schino G, Ventura R, Troisi A (2005) Grooming and aggression in captive Japanese macaques. Primates 46:207-209

Schneeberger K, Dietz M, Taborsky M (2012) Reciprocal cooperation between unrelated rats depends on cost to donor and benefit to recipient. BMC Evol Biol 12:41

Schweinfurth MK, Neuenschwander J, Engqvist L, Rentsch AK, Gygax M, Taborsky M (2017a) Do female Norway rats form social bonds? Behav Ecol Sociobiol 71:98

Schweinfurth MK, Stieger B, Taborsky M (2017b) Experimental evidence for reciprocity in allogrooming among wild-type Norway rats. Sci Rep 7:4010

Schweinfurth MK, Taborsky M (2017) The transfer of alternative tasks in reciprocal cooperation. Anim Behav 131:35-41

Seyfarth RM (1977) A model of social grooming among adult female monkeys. J Theor Biol 65:671-698

Seyfarth RM, Cheney DL (1984) Grooming, alliances and reciprocal altruism in vervet monkeys. Nature 308:541-543

Seyfarth RM, Cheney DL (2012) The evolutionary origins of friendships. Annu Rev Psychol 63:153-177

Silk JB (1982) Altruism among female macaca radiate: explanations and analysis of patterns of grooming and coalition formation. Behaviour 79:162-188

Silk JB (2002) Using the ' $F$ '-word in primatology. Behaviour 139:421-446

Silk JB, Alberts SC, Altmann J (2006) Social relationships among adult female baboons (Papio cynocephalus) II. Variation in the quality and stability of social bonds. Behav Ecol Sociobiol 61:197-204

St-Pierre A, Larose K, Dubois $F$ (2009) Long-term social bonds promote cooperation in the iterated prisoner's dilemma. Proc R Soc Lond B 276:4223-4228 
Stephens DW, McLinn CM, Stevens JR (2002) Discounting and reciprocity in an iterated prisoner's dilemma. Science 298:2216-2218

Stevens JR, Cushman FA, Hauser MD (2005) Evolving the psychological mechanisms for cooperation. Annu Rev Ecol Evol 36:499-518

Stevens JR, Hauser MD (2004) Why be nice? Psychological constraints on the evolution of cooperation. Trends Cogn Sci 8:60-65

Stopka P, Macdonald DW (1999) The market effect in the wood mouse, Apademus sylvaticus: selling information on reproductive status. Ethology 105:969-982

Taborsky M, Frommen JG, Riehl C (2016) Correlated pay-offs are key to cooperation. Philos T Roy Soc B 371:20150084

Tanaka I, Takefushi H (1993) Elimination of external parasites (lice) is the primary function of grooming in free-ranging Japanese macaques. Anthropol Sci 101:187-193

Tebbich S, Taborsky M, Winkler H (1996) Social manipulation causes cooperation in keas. Anim Behav 52:1-10

Telle HJ (1966) Beitrag zur Erkenntnis der Verhaltensweise von Ratten, vergleichend dargestellt bei Rattus norvegicus und Rattus rattus. Z Angew Zool 53:129-196

Trivers RL (1971) The evolution of reciprocal altruism. Q Rev Biol 46:35-57

Ventura R, Majolo B, Koyama NF, Hardie S, Schino G (2006) Reciprocation and interchange in wild Japanese macaques: grooming, cofeeding, and agonistic support. Am J Primatol 68:1138-1149

Viblanc VA, Mathien A, Saraux C, Viera VM, Groscolas R (2011) It costs to be clean and fit: energetics of comfort behavior in breeding-fasting penguins. PLoS ONE 6:e21110

Wilkinson GS (1984) Reciprocal food sharing in the vampire bat. Nature 308:181-184

Wilson EO (1975) Sociobiology: the new synthesis. Belknap press of Harvard University, Cambridge, MA

Wittig RM, Crockford C, Deschner T, Langergraber KE, Ziegler TE, Zuberbühler K (2014) Food sharing is linked to urinary oxytocin levels and bonding in related and unrelated wild chimpanzees. Proc $R$ Soc B 281:20133096 
Table 1 Ethogram of behaviours during the unrestricted interaction phase of the experiment.

\begin{tabular}{|c|c|c|c|c|}
\hline \begin{tabular}{|l|} 
Mode \\
Aggressive
\end{tabular} & $\begin{array}{l}\text { Behaviour } \\
\text { Threatening approach }(18.45 \%)\end{array}$ & $\begin{array}{l}\text { Description } \\
\text { Approaching rat turns its flank, head and ears } \\
\text { towards its conspecific. The legs are stretched } \\
\text { so that the stomach has no contact to the } \\
\text { ground, back is arched and fur is raised. }\end{array}$ & \begin{tabular}{|l|} 
Behaviour \\
Fighting $(1.48 \%)$ \\
\end{tabular} & $\begin{array}{l}\text { Description } \\
\text { Both rats roll over the ground } \\
\text { while holding, kicking and } \\
\text { punching each other. }\end{array}$ \\
\hline Affiliative & $\begin{array}{l}\text { Auto-grooming in close } \\
\text { proximity }(51.36 \%)\end{array}$ & $\begin{array}{l}\text { The rat cleans every part of its body that it can } \\
\text { reach. This typically starts in the front and } \\
\text { moves on to the back. The rats use their fore- } \\
\text { paws and teeth to "comb" their fur. This } \\
\text { behaviour was only classified as such when } \\
\text { performed in direct physical contact or in the } \\
\text { partner's reach ( } 2 \mathrm{~cm}) \text {. }\end{array}$ & Allogrooming (20.62\%) & $\begin{array}{l}\text { The groomer cleans the gromees } \\
\text { dorsal fur, especially in the neck } \\
\text { or facial region, by using the } \\
\text { forepaws. An allogrooming bout } \\
\text { was defined as the repeated } \\
\text { nibbling or licking of the partner's } \\
\text { body surface, except for the } \\
\text { anogenital region. A new event } \\
\text { war recorded if the grooming was } \\
\text { interrupted for at least } 10 \text { seconds. } \\
\text { Because of the preferred regions } \\
\text { for allogrooming, rats rarely } \\
\text { groom each other simultaneously. }\end{array}$ \\
\hline
\end{tabular}

Numbers in brackets denote the percentage of frequencies of the specific behaviours in relation to the total amount of all observed behaviours. The terminology and description of all behaviours and states are based on Schleif (2001) and own observations. 


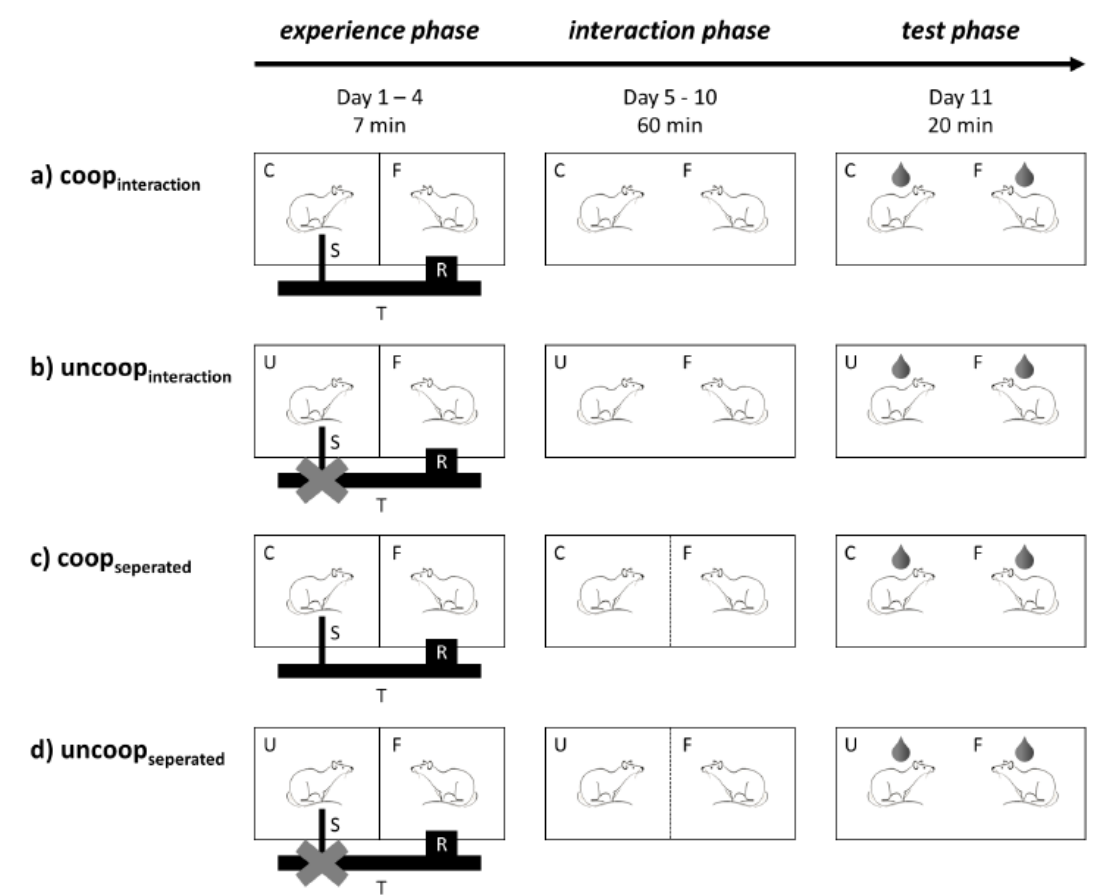

\section{Fig. 1 Experimental set up}

The experiment consisted of three phases and four treatments. All focal rats experienced all four treatments with four different partners. During the food-provisioning phase, focal rats (F) experienced on the first four days partners either being cooperative (C) by providing food ( $a$ and $c$ ) or being uncooperative $(\mathrm{U})$ by providing no food ( $\mathrm{b}$ and $\mathrm{d}$ ). Cooperative partners donated a food reward $(\mathrm{R})$ to focal rats by pulling a stick (S), which was connected to a tray $(T)$ that delivered food only to the recipient. In contrast, the uncooperative partner could not produce food to the recipient because the tray was blocked (indicated by a crossed stick). In the unrestricted interaction phase, focal individuals met cooperative or uncooperative partners for one hour on six consecutive days, while either direct interactions were allowed ( $a$ and $b$ ) or rats were prevented from physical interactions by a wire mesh barrier indicated by a dashed line (c and d). Finally, in the induced allogrooming phase, focal and partner rats experienced a saltwater application (indicated by a water drop) and we recorded allogrooming bouts during $20 \mathrm{~min}$. 
a

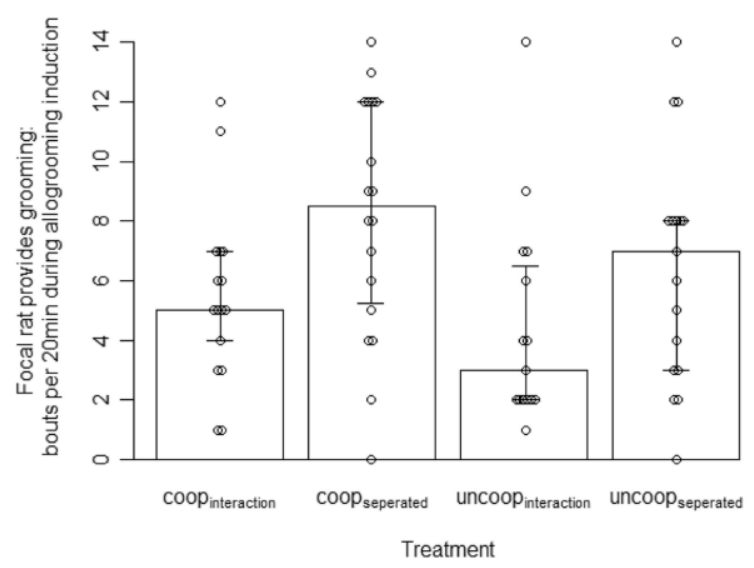

b

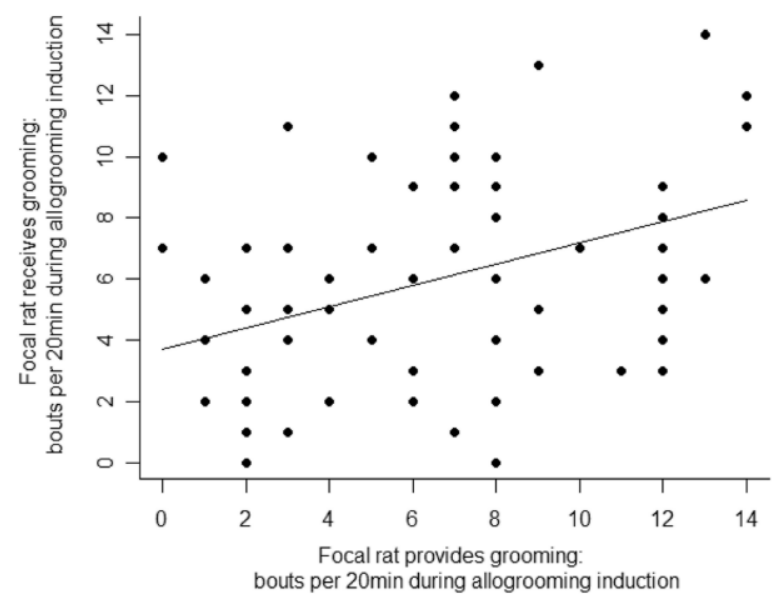

Fig. 2 Focal rat grooms partner in the induced allogrooming phase

a) Medians, interquartile ranges and individual data of grooming bouts by focal individuals are shown towards their partner. During the induced allogrooming phase, focal rats groomed cooperative food providers significantly more often than uncooperative partners (uncoop interaction $_{\text {and }}$ uncoop seperated), $_{\text {) }}$ independently of the possibility of unrestricted interactions (coop interaction $_{\text {and }}$ coop $_{\text {seperated }}$ ). Focal rats also groomed those partners more often, with which they previously could not interact, independently of whether the partner previously provided food or not ( $\operatorname{coop}_{\text {seperated }}$ and uncoop $\mathrm{p}_{\text {seperated }}$ ). b) In the induced allogrooming phase, received grooming bouts correlated positively with given grooming bouts by focal individuals. 

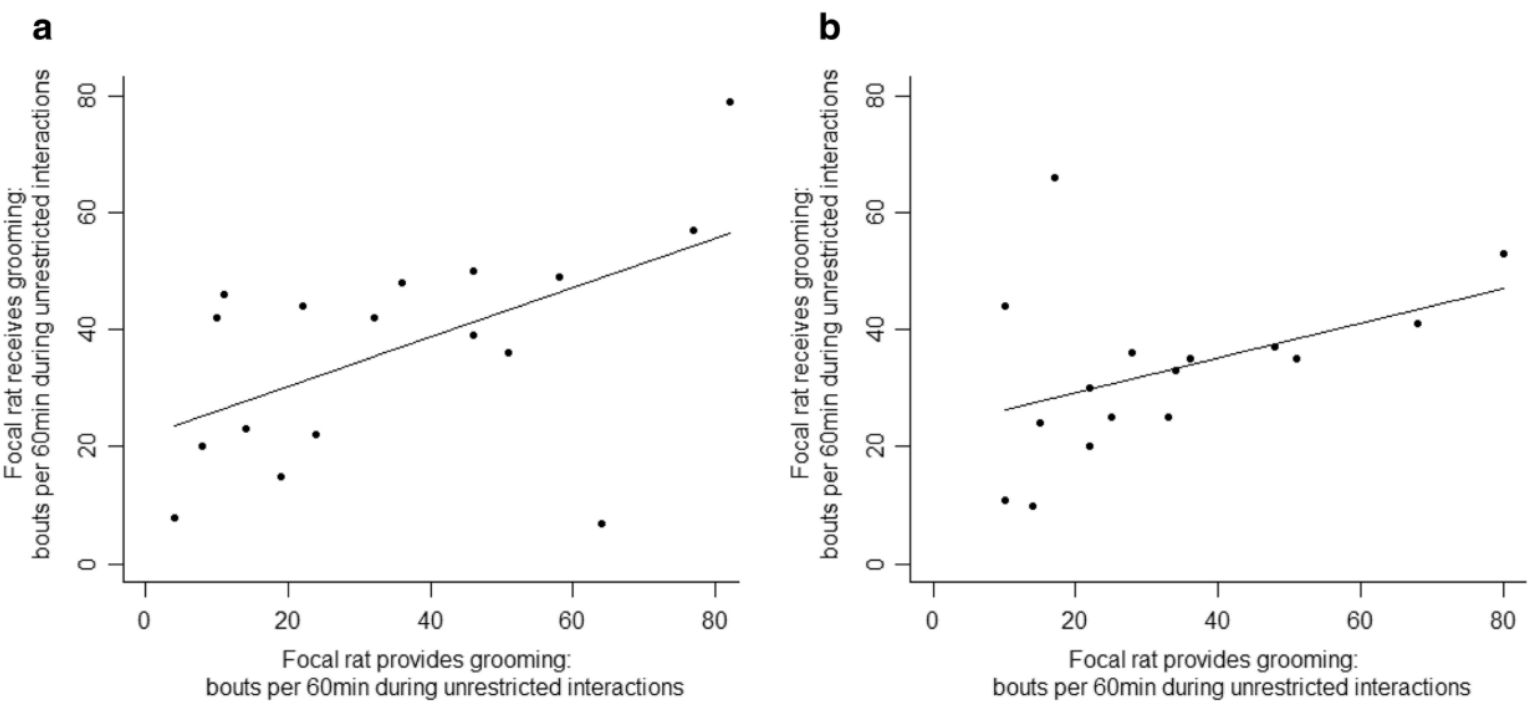

Fig. 3 Focal rat grooms partner in the unrestricted interaction phase

During unrestricted interactions, grooming rates by focal individuals correlated positively with received grooming by their previously experienced cooperative a) and uncooperative b) partners. 
a

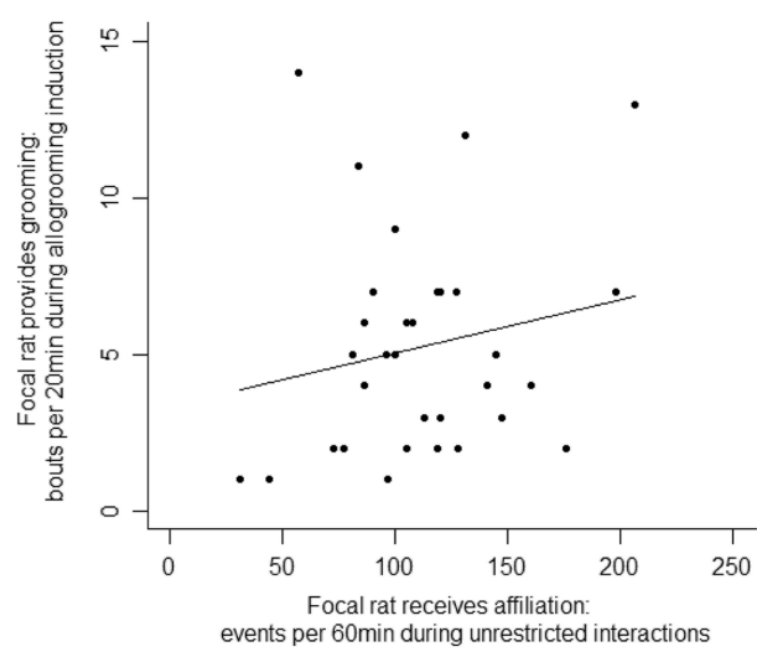

b

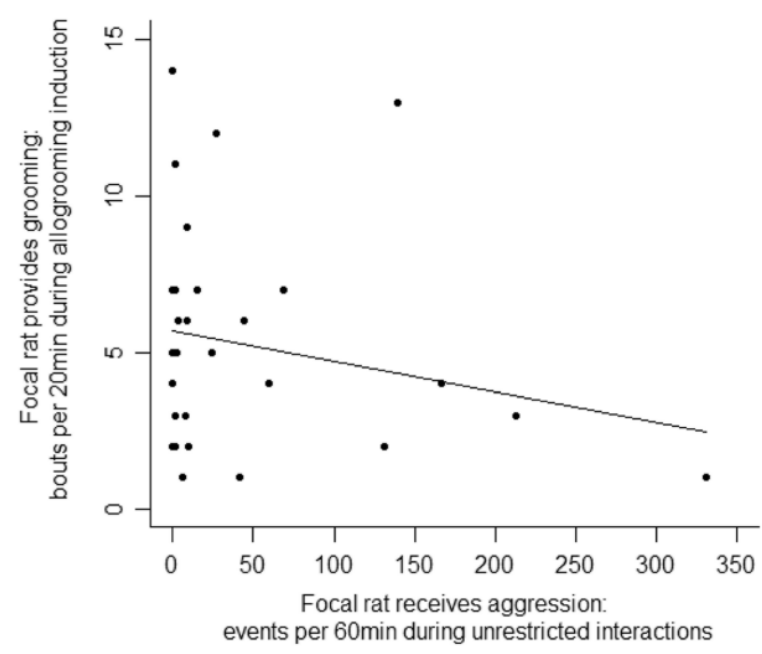

Fig. 4 Influence of affiliative and aggressive behaviours on focal rat grooming behaviour in the induced allogrooming phase

Experienced affiliative behaviours during the unrestricted interaction phase tended to increase the number of grooming bouts provided by focal individuals in the induced allogrooming phase a) whereas experienced aggression during the unrestricted interaction phase decreased the number of grooming bouts by focal individuals in the induced allogrooming phase $b$ ). 